\title{
When is the Uvarov transformation positive definite?
}

\author{
Matthias Humet \\ Marc Van Barel \\ Report TW 593, April 2011
}

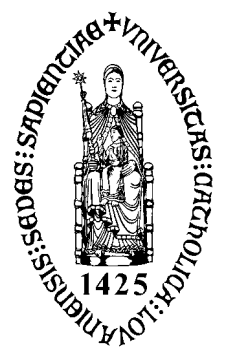

Katholieke Universiteit Leuven Department of Computer Science 


\title{
When is the Uvarov transformation positive definite?
}

\author{
Matthias Humet \\ Marc Van Barel \\ Report TW 593, April 2011
}

Department of Computer Science, K.U.Leuven

\begin{abstract}
Let $\mathcal{L}$ be a positive definite bilinear functional, then the Uvarov transformation of $\mathcal{L}$ is given by $\mathcal{U}(p, q)=\mathcal{L}(p, q)+m p(\alpha) \bar{q}\left(\alpha^{-1}\right)+\bar{m} p\left(\bar{\alpha}^{-1}\right) \bar{q}(\bar{\alpha})$ where $|\alpha|>1, m \in \mathbb{C}$. In this paper we analyze conditions on $m$ for $\mathcal{U}$ to be positive definite in the linear space of polynomials of degree less than or equal to $n$. In particular, we show that $m$ has to lie inside a circle in the complex plane defined by $\alpha, n$ and the moments associated with $\mathcal{L}$. We also give an upper bound for the radius of this circle that depends only on $\alpha$ and $n$. This and other conditions on $m$ are visualized for some examples.
\end{abstract}

Keywords : linear functional, spectral transformation, Toeplitz matrix, positive definite.

MSC : Primary : 30E05, Secondary : 15A63. 
Numerical Algorithms manuscript No.

(will be inserted by the editor)

\title{
When is the Uvarov transformation positive definite?
}

\author{
Matthias Humet • Marc Van Barel
}

Received: date / Accepted: date

Abstract Let $\mathcal{L}$ be a positive definite bilinear functional, then the Uvarov transformation of $\mathcal{L}$ is given by $\mathcal{U}(p, q)=\mathcal{L}(p, q)+m p(\alpha) \bar{q}\left(\alpha^{-1}\right)$

$+\bar{m} p\left(\bar{\alpha}^{-1}\right) \bar{q}(\bar{\alpha})$ where $|\alpha|>1, m \in \mathbb{C}$. In this paper we analyze conditions on $m$ for $\mathcal{U}$ to be positive definite in the linear space of polynomials of degree less than or equal to $n$. In particular, we show that $m$ has to lie inside a circle in the complex plane defined by $\alpha, n$ and the moments associated with $\mathcal{L}$. We also give an upper bound for the radius of this circle that depends only on $\alpha$ and $n$. This and other conditions on $m$ are visualized for some examples.

Keywords linear functional · spectral transformation · Toeplitz matrix · positive definite

Mathematics Subject Classification (2000) 30E05 $15 \mathrm{~A} 63$

\section{Introduction}

Let $\mathbb{L}_{m, n}, m \leq n$, be the vector space of Laurent polynomials

$$
p(z)=\sum_{k=m}^{n} a_{k} z^{k}, \quad a_{k} \in \mathbb{C} .
$$

The research was partially supported by the Research Council K.U.Leuven, project OT/10/038 (Multi-parameter model order reduction and its applications), CoE EF/05/006 Optimization in Engineering (OPTEC), and by the Interuniversity Attraction Poles Programme, initiated by the Belgian State, Science Policy Office, Belgian Network DYSCO (Dynamical Systems, Control, and Optimization).

M. Humet · M. Van Barel

Department of Computer Science, Katholieke Universiteit Leuven

Celestijnenlaan 200a - bus 2402

3001 Heverlee

Tel.: +32-16-327563

Fax: +32-16-327996

E-mail: matthias.humet@cs.kuleuven.be, marc.vanbarel@cs.kuleuven.be 
We denote by $\mathbb{P}=\mathbb{L}_{0, \infty}$ the vector space of polynomials with complex coefficients and by $\mathbb{P}_{n}=\mathbb{L}_{0, n}$ its subspace with polynomials of degree less than or equal to $n$.

Next we consider a bilinear functional $\mathcal{L}$ defined on $\mathbb{P}_{n}$, which is Hermitian, $\overline{\mathcal{L}(p, q)}=\mathcal{L}(q, p)$, and unitary, $\mathcal{L}(z p, z q)=\mathcal{L}(p, q)$. The moment matrix $T_{n} \in$ $\mathbb{C}^{(n+1) \times(n+1)}$ associated with $\mathcal{L}$ is the Toeplitz matrix

$$
T_{n}=\left[\mathcal{L}\left(z^{i}, z^{j}\right)\right]_{i, j=0}^{n}=\left(\begin{array}{ccccc}
c_{0} & \overline{c_{1}} & \ldots & \overline{c_{n-1}} & \overline{c_{n}} \\
c_{1} & c_{0} & \ddots & & \overline{c_{n-1}} \\
\vdots & \ddots & \ddots & \ddots & \vdots \\
c_{n-1} & & \ddots & c_{0} & \overline{c_{1}} \\
c_{n} & c_{n-1} & \ldots & c_{1} & c_{0}
\end{array}\right)
$$

where $c_{k}=\mathcal{L}\left(z^{k}, 1\right), k=0, \ldots, n$, are the moments associated with $\mathcal{L}$. We will assume that $c_{0}=1$.

Definition 1 [7]

(i) $\mathcal{L}$ is quasi-definite on $\mathbb{P}_{n}$ if $T_{n}$ is strongly regular, i.e., if all the leading principal submatrices of $T_{n}$ are nonsingular.

(ii) $\mathcal{L}$ is positive definite on $\mathbb{P}_{n}$ if $T_{n}>0$, i.e., if $T_{n}$ is positive definite.

If $\mathbb{P}_{n}=\mathbb{P}$, we will simply say that $\mathcal{L}$ is quasi-definite (or positive definite). Note that (ii) is equivalent to $\mathcal{L}(p, p)>0$ for all $p \in \mathbb{P}_{n}, p \neq 0$.

$\mathcal{L}$ can be written as $\mathcal{L}(p, q)=\mathcal{F}\left(p(z) \bar{q}\left(\frac{1}{z}\right)\right)$, where $\mathcal{F}$ is a linear functional defined on $\mathbb{L}_{-n, n}$ with

$$
\mathcal{F}\left(z^{k}\right)= \begin{cases}c_{k} & k \geq 0 \\ \overline{c_{-k}} & k \leq 0\end{cases}
$$

The bar on $\bar{q}(z)$ denotes complex conjugation of the coefficients of $q(z)$. It is well known that if $\mathcal{L}$ is positive definite, then it has an integral representation given by

$$
\mathcal{L}(p, q)=\int_{\mathbb{T}} p(z) \bar{q}\left(\frac{1}{z}\right) d \mu(z)
$$

where $d \mu(z)$ is a positive measure on the unit circle $\mathbb{T}=\{z \in \mathbb{C}:|z|=1\}$ (see $[6,4,5])$.

In this paper we focus our attention to the following linear spectral transformation of $\mathcal{L}$, called the Uvarov transformation

$$
\mathcal{U}(p, q)=\mathcal{L}(p, q)+m p(\alpha) \bar{q}\left(\frac{1}{\alpha}\right)+\bar{m} p\left(\frac{1}{\bar{\alpha}}\right) \bar{q}(\bar{\alpha}), \quad m \in \mathbb{C},|\alpha|>1
$$

A recent overview of linear spectral transformations is given in [1], both for the real line and for the unit circle. Conditions for quasi-definiteness and positive definiteness of $\mathcal{U}$ in terms of the orthogonal polynomials associated with $\mathcal{L}$ have been studied in $[8,2]$. In this paper, we deduce a necessary condition for positive definiteness of $\mathcal{U}$ in terms of the moments $c_{k}$ associated with $\mathcal{L}$. 
One of the reasons for studying this transformation is that it appears naturally when considering the Geronimus transformation (see $[8,3,1]$ ), defined by

$$
\mathcal{G}((z-\alpha) p(z),(z-\alpha) q(z))=\mathcal{L}(p, q), \quad|\alpha|>1 .
$$

Indeed, it is proven in [8] that for a positive definite functional $\mathcal{L}$ given by $(2)$, the Geronimus transformation defined by (4) satisfies

$$
\mathcal{G}(p, q)=\mathcal{L}\left(\frac{p(z)}{z-\alpha}, \frac{q(z)}{z-\alpha}\right)+m p(\alpha) \bar{q}\left(\frac{1}{\alpha}\right)+\bar{m} p\left(\frac{1}{\bar{\alpha}}\right) \bar{q}(\bar{\alpha})
$$

where $m \in \mathbb{C}$ is a free parameter.

In Section 2 we show that for a positive definite $\mathcal{L}$ and for $|\alpha|>1$, if the Uvarov transformation $\mathcal{U}$ is positive definite on $\mathbb{P}_{n}$, then $m$ lies inside a circle in the complex plane. We derive an upper bound for the radius of this circle in Section 3, which depends only on $n$ and $\alpha$. By showing that this upper bound goes to zero as $n \rightarrow \infty$, we prove that $\mathcal{U}$ is positive definite on $\mathbb{P}$ iff $m=0$.

Finally, in Section 4 we illustrate the fact that we only have a necessary condition for $\mathcal{U}$ to be positive definite on $\mathbb{P}_{n}$. Moreover, we show that the upper bound on the radius of the circle can be much too conservative and that although it is a monotonically decreasing function of $n$, the radius does not have to be.

\section{Uvarov moment matrix}

We assume that $\mathcal{L}$ is positive definite, so $T_{k}>0$ for all $k \geq 0$, and we assume that $c_{0}=\mathcal{L}(1,1)=1$. If $c_{k}=\mathcal{L}\left(z^{k}, 1\right)$, then the moments $d_{k}=\mathcal{U}\left(z^{k}, 1\right)$ are given by

$$
d_{k}=c_{k}+m \alpha^{k}+\bar{m} \frac{1}{\bar{\alpha}^{k}} .
$$

Let $T=T_{n-1}$, then the moment matrix $T_{\mathcal{U}} \in \mathbb{R}^{n \times n}$ associated with $\mathcal{U}$ can be written as

$$
T_{\mathcal{U}}=T+m \boldsymbol{u} \boldsymbol{v}^{*}+\bar{m} \boldsymbol{v} \boldsymbol{u}^{*},
$$

or if $m=x+i y$ we have

$$
T_{\mathcal{U}}=T+x\left(\boldsymbol{u} \boldsymbol{v}^{*}+\boldsymbol{v} \boldsymbol{u}^{*}\right)+i y\left(\boldsymbol{u v ^ { * }}-\boldsymbol{v} \boldsymbol{u}^{*}\right)
$$

where

$$
\begin{aligned}
& \boldsymbol{u}=\left[\begin{array}{llll}
1 & \alpha & \cdots & \alpha^{n-1}
\end{array}\right]^{T}, \\
& \boldsymbol{v}=\left[\begin{array}{llll}
1 & \frac{1}{\bar{\alpha}} & \cdots & \frac{1}{\overline{\alpha^{n-1}}}
\end{array}\right]^{T} .
\end{aligned}
$$

In this section we will show that if $T_{\mathcal{U}}(m)>0$, then $m$ must lie inside a circle. This condition is necessary but not sufficient. Indeed, the circle will contain the convex set $\mathcal{D}$ of the following proposition. 
Proposition 1 Let $\mathcal{D}=\left\{m \in \mathbb{C}: T_{\mathcal{U}}(m)>0\right\}$ then $\mathcal{D}$ is a convex set containing zero.

Proof If $T_{\mathcal{U}}\left(m_{1}\right)>0$ and $T_{\mathcal{U}}\left(m_{2}\right)>0$, then $T_{\mathcal{U}}\left((1-\kappa) m_{1}+\kappa m_{2}\right)>0$ for all $\kappa \in[0,1]$. Moreover $T_{\mathcal{U}}(0)=T>0$.

Let $U=\left[\begin{array}{ll}\boldsymbol{u} & \boldsymbol{v}\end{array}\right]$. Since $|\alpha|>1, \boldsymbol{u}$ and $\boldsymbol{v}$ are linearly independent. It follows that if the matrix $T_{\mathcal{U}}>0$, then $U^{*} T_{\mathcal{U}} U>0$, i.e.,

$$
U^{*} T U+x\left(U^{*} \boldsymbol{u} \boldsymbol{v}^{*} U+U^{*} \boldsymbol{v} \boldsymbol{u}^{*} U\right)+i y\left(U^{*} \boldsymbol{u} \boldsymbol{v}^{*} U-U^{*} \boldsymbol{v} \boldsymbol{u}^{*} U\right)>0 .
$$

Note that this is a necessary condition for $T_{\mathcal{U}}>0$, but not a sufficient one. We will illustrate this fact in Section 4 with some numerical experiments. We introduce some notation and note the following:

$$
\begin{aligned}
\theta_{u} & \equiv \boldsymbol{u}^{*} \boldsymbol{u}=\sum_{i=0}^{n-1}|\alpha|^{2 i}=\frac{|\alpha|^{2 n}-1}{|\alpha|^{2}-1}, \\
\theta_{v} & \equiv \boldsymbol{v}^{*} \boldsymbol{v}=\sum_{i=0}^{n-1}|\alpha|^{-2 i}=\frac{|\alpha|^{-2 n}-1}{|\alpha|^{-2}-1}=\frac{1}{|\alpha|^{2 n-2}} \theta_{u}, \\
\boldsymbol{u}^{*} \boldsymbol{v} & =\boldsymbol{v}^{*} \boldsymbol{u}=n, \\
\left(\begin{array}{cc}
t_{1} & \overline{t_{2}} \\
t_{2} & t_{3}
\end{array}\right) & \equiv U^{*} T U .
\end{aligned}
$$

After some straightforward calculations, condition (7) becomes

$$
\left(\begin{array}{l}
t_{1} \overline{t_{2}} \\
t_{2} t_{3}
\end{array}\right)+x\left(\begin{array}{cc}
2 n \theta_{u} & \theta_{u} \theta_{v}+n^{2} \\
\theta_{u} \theta_{v}+n^{2} & 2 n \theta_{v}
\end{array}\right)+i y\left(\begin{array}{cc}
0 & \theta_{u} \theta_{v}-n^{2} \\
n^{2}-\theta_{u} \theta_{v} & 0
\end{array}\right)>0 .
$$

Positive definiteness is equivalent to having positive leading principal minors, so we get conditions

$$
x>-\frac{t_{1}}{2 n \theta_{u}}
$$

and

$$
\begin{aligned}
&\left(t_{1}+2 n \theta_{u} x\right)\left(t_{3}+2 n \theta_{v} x\right)-\left(t_{2}+x\left(n^{2}+\theta_{u} \theta_{v}\right)+i y\left(n^{2}-\theta_{u} \theta_{v}\right)\right) \\
& \cdot\left(\overline{t_{2}}+x\left(n^{2}+\theta_{u} \theta_{v}\right)-i y\left(n^{2}-\theta_{u} \theta_{v}\right)\right)>0 .
\end{aligned}
$$

If we further elaborate the latter expression, putting together terms with equal powers of $x$ and $y$, we get

$$
\begin{gathered}
x^{2}+y^{2}+x \frac{2 \Re\left(t_{2}\right)\left(\theta_{u} \theta_{v}+n^{2}\right)-2 n\left(t_{1} \theta_{v}+t_{3} \theta_{u}\right)}{\left(n^{2}-\theta_{u} \theta_{v}\right)^{2}} \\
+y \frac{2 \Im\left(t_{2}\right)}{n^{2}-\theta_{u} \theta_{v}}+\frac{\left|t_{2}\right|^{2}-t_{1} t_{3}}{\left(n^{2}-\theta_{u} \theta_{v}\right)^{2}}<0,
\end{gathered}
$$


where $\Re\left(t_{2}\right)$ and $\Im\left(t_{2}\right)$ denote the real and imaginary part of $t_{2}$, respectively. Note that (12) means that the point $(x, y)$ has to lie inside a circle. In the following section, we prove that $\theta_{u} \theta_{v}-n^{2} \neq 0$ for $n>1$.

In order to check that (10) is always satisfied if (11) or (12) holds, just note that if $x=-\frac{t_{1}}{2 n \theta_{u}}<0$, condition (11) can never be satisfied. Moreover the point $(x, y)=(0,0)$ always satisfies (12), so the circle defined by (12) lies on the right of the line defined by (10). We conclude that every point inside the circle (12), satisfies (10).

\section{Upper bound on the radius}

The radius $R$ of a circle defined by $x^{2}+y^{2}+2 a x+2 b y+c=0$ satisfies $R^{2}=-c+a^{2}+b^{2}$, so for the circle defined by (12) we can find the following expression for the radius:

$$
R^{2}=\frac{t_{1} t_{3}-\Re\left(t_{2}\right)^{2}}{\left(\theta_{u} \theta_{v}-n^{2}\right)^{2}}+\frac{\left(\Re\left(t_{2}\right)\left(\theta_{u} \theta_{v}+n^{2}\right)-n\left(t_{1} \theta_{v}+t_{3} \theta_{u}\right)\right)^{2}}{\left(\theta_{u} \theta_{v}-n^{2}\right)^{4}} .
$$

We can further simplify this expression as follows. Let $P \in \mathbb{R}^{n \times n}$ be the matrix with zeros everywhere, except for its $n$-th antidiagonal where it has ones, i.e.,

$$
P_{i, j}= \begin{cases}1 & \text { if } i+j=n+1 \\ 0 & \text { otherwise }\end{cases}
$$

Next we note that

$$
\boldsymbol{u}=\alpha^{n-1} P \overline{\boldsymbol{v}} \text { and } P T^{T} P=T .
$$

from (6) and because $T$ is Toeplitz. Hence, from (9), we have

$$
t_{1}=\boldsymbol{u}^{*} T \boldsymbol{u}=\boldsymbol{u}^{T} T^{T} \overline{\boldsymbol{u}}=|\alpha|^{2 n-2} \boldsymbol{v}^{*} P T^{T} P \boldsymbol{v}=|\alpha|^{2 n-2} \boldsymbol{v}^{*} T \boldsymbol{v}=|\alpha|^{2 n-2} t_{3} .
$$

so it follows from (8) that $t_{1} \theta_{v}=t_{3} \theta_{u}$.

We put this into (13) and after some straightforward computations we get

$$
\begin{aligned}
R^{2}= & \frac{t_{1} t_{3}}{\left(\theta_{u} \theta_{v}-n^{2}\right)^{2}}+4 \frac{\left(n^{2} \Re\left(t_{2}\right)-n t_{1} \theta_{v}\right) \Re\left(t_{2}\right)\left(\theta_{u} \theta_{v}-n^{2}\right)}{\left(\theta_{u} \theta_{v}-n^{2}\right)^{4}} \\
& \quad+4 \frac{\left(n^{2} \Re\left(t_{2}\right)-n t_{1} \theta_{v}\right)^{2}}{\left(\theta_{u} \theta_{v}-n^{2}\right)^{4}} . \\
= & \frac{t_{1} t_{3}}{\left(\theta_{u} \theta_{v}-n^{2}\right)^{2}}+\frac{4}{\left(\theta_{u} \theta_{v}-n^{2}\right)^{4}} \\
& \cdot\left(n^{2} \Re\left(t_{2}\right)^{2} \theta_{u} \theta_{v}+n^{2} t_{1}^{2} \theta_{v}^{2}-n t_{1} \theta_{v} \Re\left(t_{2}\right) \theta_{u} \theta_{v}-n^{3} t_{1} \theta_{v} \Re\left(t_{2}\right)\right) \\
= & \frac{1}{\left(1-\frac{n^{2}}{\theta_{u} \theta_{v}}\right)^{4}}\left(\frac{t_{1} t_{3} n^{4}}{\left(\theta_{u} \theta_{v}\right)^{4}}+\frac{t_{1} t_{3}}{\left(\theta_{u} \theta_{v}\right)^{2}}+2 \frac{t_{1} t_{3} n^{2}}{\left(\theta_{u} \theta_{v}\right)^{3}}\right. \\
& \left.\quad+4 \frac{\Re\left(t_{2}\right)^{2} n^{2}}{\left(\theta_{u} \theta_{v}\right)^{3}}-4 \frac{t_{1} \theta_{v} \Re\left(t_{2}\right) n}{\left(\theta_{u} \theta_{v}\right)^{3}}-4 \frac{t_{1} \theta_{v} \Re\left(t_{2}\right) n^{3}}{\left(\theta_{u} \theta_{v}\right)^{4}}\right) .
\end{aligned}
$$


An upper bound for $R^{2}$ can be found by noting that $\Re\left(t_{2}\right)^{2}<t_{1} t_{3}$ from (9) and

$$
\begin{aligned}
& t_{1}=\boldsymbol{u}^{*} T \boldsymbol{u} \leq \theta_{u} \lambda_{\max }(T)<\theta_{u} n, \\
& t_{3}=\boldsymbol{v}^{*} T \boldsymbol{v} \leq \theta_{v} \lambda_{\max }(T)<\theta_{v} n,
\end{aligned}
$$

since $\lambda_{\max }(T)<\operatorname{trace}(T)=n$, because we assume that $c_{0}=1 .{ }^{1}$ We get

$$
\begin{aligned}
R^{2}<\frac{1}{\left(1-\frac{n^{2}}{\theta_{u} \theta_{v}}\right)^{4}}\left(\frac{n^{6}}{\left(\theta_{u} \theta_{v}\right)^{3}}+\right. & \frac{n^{2}}{\theta_{u} \theta_{v}}+2 \frac{n^{4}}{\left(\theta_{u} \theta_{v}\right)^{2}} \\
& \left.+4 \frac{n^{4}}{\left(\theta_{u} \theta_{v}\right)^{2}}+4 \frac{n^{3}}{\left(\theta_{u} \theta_{v}\right)^{1.5}}+4 \frac{n^{5}}{\left(\theta_{u} \theta_{v}\right)^{2.5}}\right) .
\end{aligned}
$$

We now prove that this upper bound is a decreasing function of $n$ and that it goes to zero asymptotically for $n \rightarrow \infty$. Let $q \equiv|\alpha|^{2}>1$, then (for $n>1$ )

$$
\begin{aligned}
\theta_{u} \theta_{v} & =\sum_{i=1}^{n} q^{i} \sum_{j=1}^{n} q^{-j}=\sum_{i, j=1}^{n} q^{i-j}=n+\sum_{\substack{i, j=1 \\
i<j}}^{n}\left(q^{i-j}+q^{j-i}\right) \\
& >n+2 \sum_{\substack{i, j=1 \\
i<j}}^{n} 1=n^{2},
\end{aligned}
$$

since for any $r \in \mathbb{R}^{+}, r \neq 1: r+r^{-1}>2$. Hence,

$$
\frac{n^{2}}{\theta_{u} \theta_{v}}<1
$$

Note that

$$
\theta_{u} \theta_{v}=\frac{q^{n}+q^{-n}-2}{q+q^{-1}-2}
$$

from which it follows that

$$
\lim _{n \rightarrow \infty} \frac{n^{2}}{\theta_{u} \theta_{v}}=0 .
$$

In addition to going asymptotically to zero, the function $\frac{n^{2}}{\theta_{u} \theta_{v}}$ is also strictly decreasing, because the derivative of its inverse is positive. Indeed,

$$
\begin{aligned}
\frac{d}{d n}\left(\frac{\theta_{u} \theta_{v}}{n^{2}}\right) & =\frac{\zeta^{-1}\left(q^{n}-q^{-n}\right) n^{2}-\zeta^{-1}\left(q^{n}+q^{-n}-2\right) 2 n}{n^{4}} \\
& =\frac{(n-2)\left(q^{n}-q^{-n}\right)+4-4 q^{-n}}{\zeta n^{3}}>0, \quad n \geq 2,
\end{aligned}
$$

where $\zeta=q+q^{-1}-2$. Hence the upper bound (15) for $R^{2}$ is strictly decreasing and it follows from (16) that it goes to zero for $n \rightarrow \infty$. From this last statement, we conclude that $T_{\mathcal{U}}>0$ on $\mathbb{P}$ iff $m=0$.

1 Note that the inequality holds (strictly) because $T>0$. 


\section{Numerical experiments}

In this section we consider some examples of positive definite Toeplitz matrices $T \in \mathbb{C}^{n \times n}$ and we compare the circle of condition (7) with the convex set $\mathcal{D}$ of Proposition 1. In addition, we show that the upper bound for the radius $R$ in (15) can be much too conservative and that although the upper bound for the radius $R$ is monotonically decreasing, the radius $R$ not necessarily is.

Consider the Toeplitz matrices

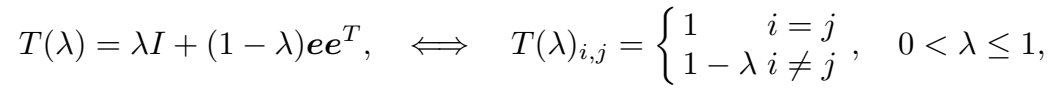

with $I$ the $n \times n$ identity matrix and $\boldsymbol{e}=\left[\begin{array}{llll}1 & 1 & \cdots & 1\end{array}\right]^{T}$. For certain values of $\lambda$ and $\alpha$, we compute the circle of (7) and the border of the convex set $\mathcal{D}$. We use $n=100$.

The border of $\mathcal{D}$ is computed as follows. For a wide range of angles of $m$, a bisection algorithm finds two values for the modulus of $m$, with $T_{\mathcal{U}}>0$ for the smaller value and $T_{\mathcal{U}} \leq 0$ for the larger value. The relative distance $\epsilon$ between the values determines the precision of the border and un upper bound for $\epsilon$ is a parameter for the bisection algorithm. We use $\epsilon<1 e-7$ and we use the mean of both values as modulus for every angle of $m$.

In Figure 1, we give an example of cases where the convex set $\mathcal{D}$ does not agree with the circle condition (7). We do this for four values of $\lambda$ and with $\alpha=1.001 e^{i 0.03}$. When $\lambda=1$, or when $T$ is equal to the identity matrix, we see that the convex set lies on top of the circle. This agrees with the fact that if $T=I$, then (7) becomes a sufficient condition. For smaller values of $\lambda$, this is not true anymore.

Note that the scaling varies among the subfigures of Figure 1, so the circles have different sizes. Figure 2 shows the circles and convex sets separately. Note that the behaviour in function of $\lambda$ seems different.

Figure 3 is a plot of the radius $R$ of equation (13) for the same four matrices and of the upper bound (15), both as function of $n$. Note that the upper bound is many orders of magnitude larger than the radii. However, for $\alpha=2 e^{i \frac{\pi}{3}}$, the exponential term $|\alpha|^{2 n}$ dominates much more in both the radius (14) and the upper bound (15) and the difference is a lot smaller, as can be seen in Figure 4 . We only show the case $\lambda=1$, because the difference with the other cases is not visually noticeable. Finally, we show in Figure 5 that although the upper bound of the radius is strictly decreasing, the radius does not need to be. Figure 5 shows the radius as a function of $n$ for $\lambda=0.001$ and $\alpha=1.001 e^{i \frac{\pi}{3}}$.

\section{Conclusions}

In this paper, we proved that the Uvarov transformation $\mathcal{U}$ of a positive definite functional $\mathcal{L}$, given by $(3)$, is positive definite on $\mathbb{P}$ iff $m=0$. When we restrict positive definitess to the space $\mathbb{P}_{n}$, we obtained the necessary condition (12) that $m$ has to lie in a circle. It is however sufficient for $m$ to lie in the convex 

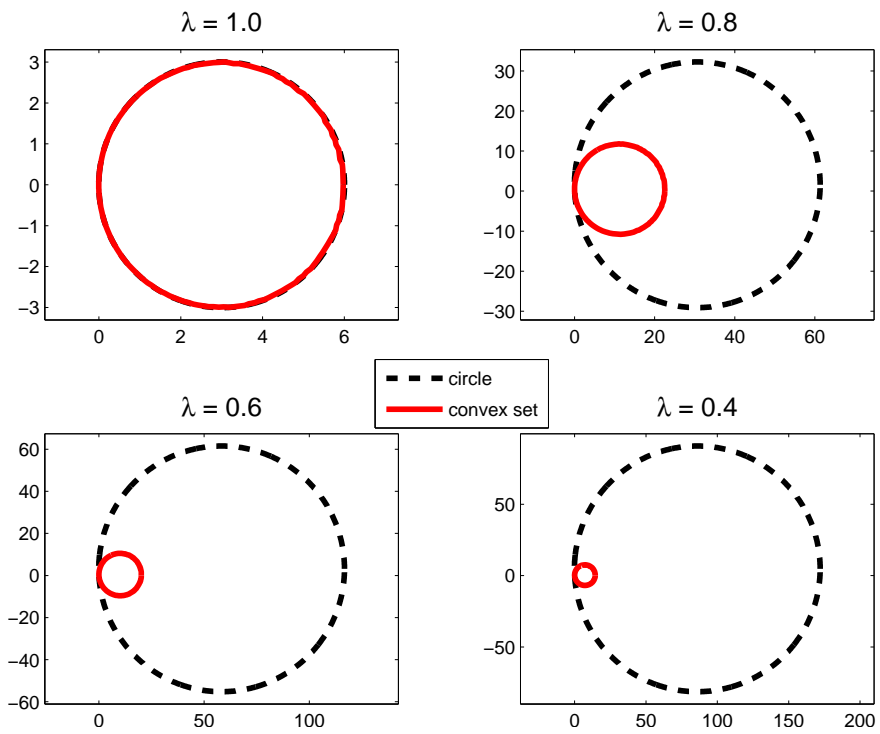

Figure 1 The circle of (7) and the border of the convex set $\mathcal{D}$, for some values of $\lambda$.
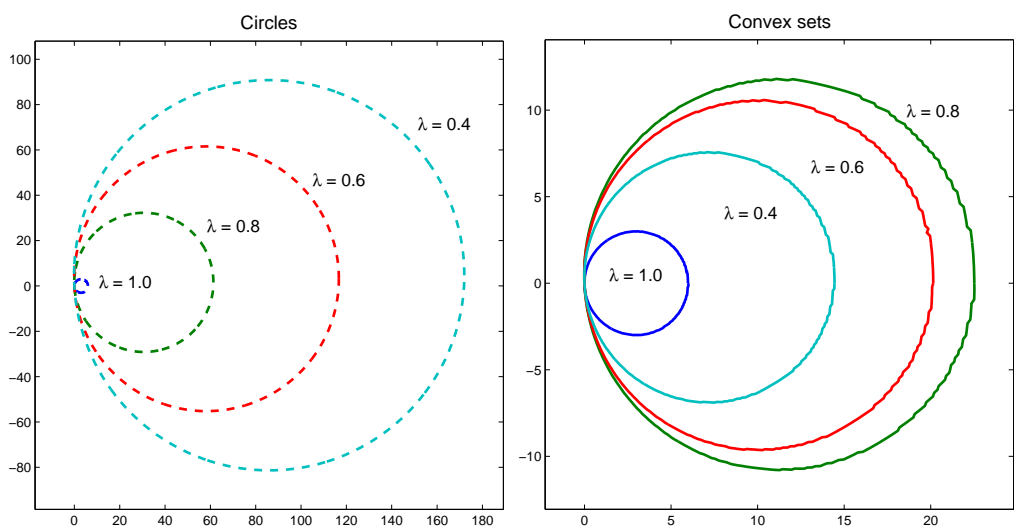

Figure 2 The circle of (7) and the border of the convex set $\mathcal{D}$, for some values of $\lambda$.

set $\mathcal{D}$ of Proposition 1, which can be smaller than this circle. We also obtained an upper bound for the radius of this circle, which depends only on $\alpha$ and $n$. This upper bound can be very conservative and even though it is strictly decreasing, the radius does not need to be.

We believe that these results can play an important role in the behaviour of algorithms to compute the Verblunsky coefficients associated with the Geronimus transformation (see Introduction and [3]). These algorithms will be discussed in a following paper. 


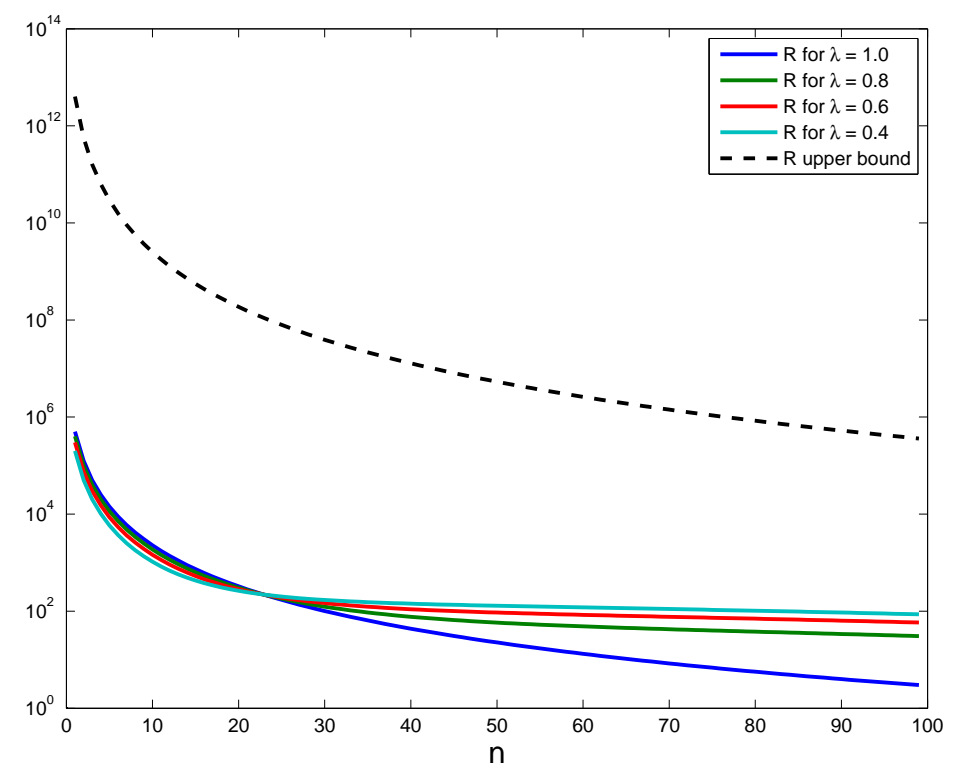

Figure 3 Semilog plot of the radii in function of $n$ for different values of $\lambda$ and $\alpha=$ $1.001 e^{i 0.03}$, and of the upper bound (15) for the radius

\section{References}

1. Castillo, K., Garza, L., Marcellán, F.: Linear spectral transformations, Hessenberg matrices, and orthogonal polynomials. Rend. Circ. Mat. Palermo 82, 3-26 (2010)

2. Daruis, L., Hernández, J., Marcellán, F.: Spectral transformations for Hermitian Toeplitz matrices. J. Comput. Appl. Math. 202(2), 155-176 (2007)

3. Garza, L., Hernández, J., Marcellán, F.: Orthogonal polynomials and measures on the unit circle. The Geronimus transformations. J. Comput. Appl. Math. 233(5), 1220-1231 (2010)

4. Geronimus, Y.L.: Polynomials orthogonal on a circle and their applications. American Mathematical Society, Providence, RI (1954)

5. Grenander, U., Szegö, G.: Toeplitz forms and their applications, 2nd edition edn. University of California Press, Berkely (1958)

6. Hernández, J., Marcellán, F.: Christoffel transforms and Hermitian linear functionals. Med. J. Math. 2(4), 451-458 (2005)

7. Jones, W.B., Njåstad, O., Thron, W.J.: Moment theory, orthogonal polynomials, quadrature, and continued fractions associated with the unit circle. blms 21, 113-152 (1989)

8. Marcellán, F.: Polinomios ortogonales no estándar. Aplicaciones en análisis numérico y teoría de la aproximación. Rev. Acad. Colombiana de Cienc. Exact., Fís. y Natur. 30(117), 563-579 (2006). In Spanish 


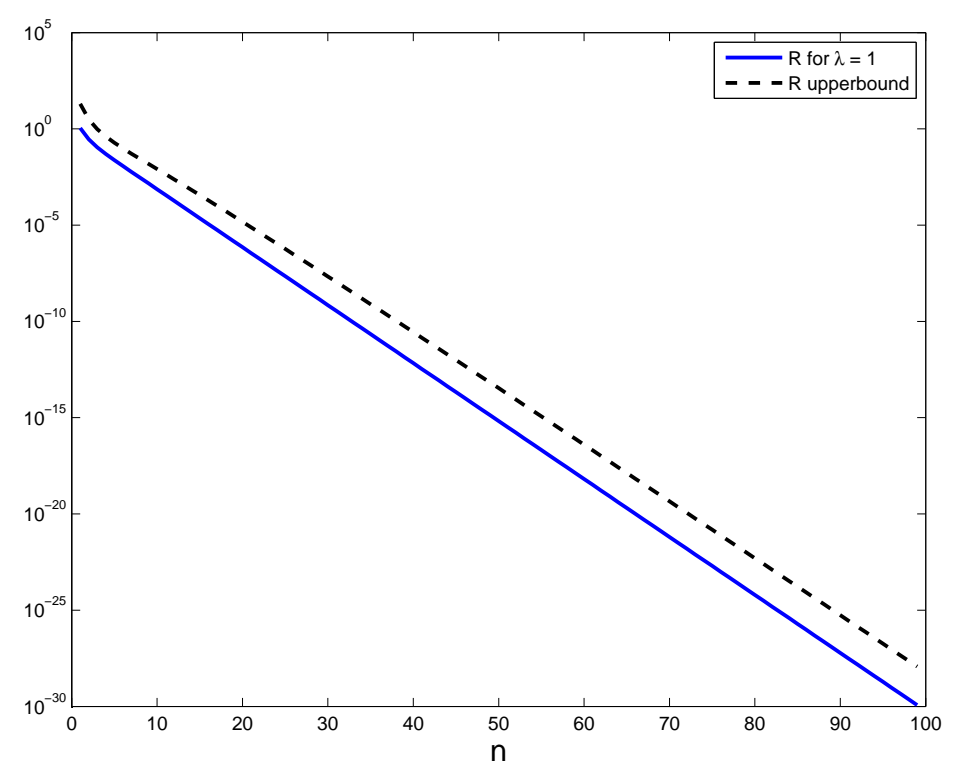

Figure 4 Semilog plot of the radius in function of $n$ for $\lambda=1$ and $\alpha=2 e^{i \frac{\pi}{3}}$, and of the upper bound (15) for the radius

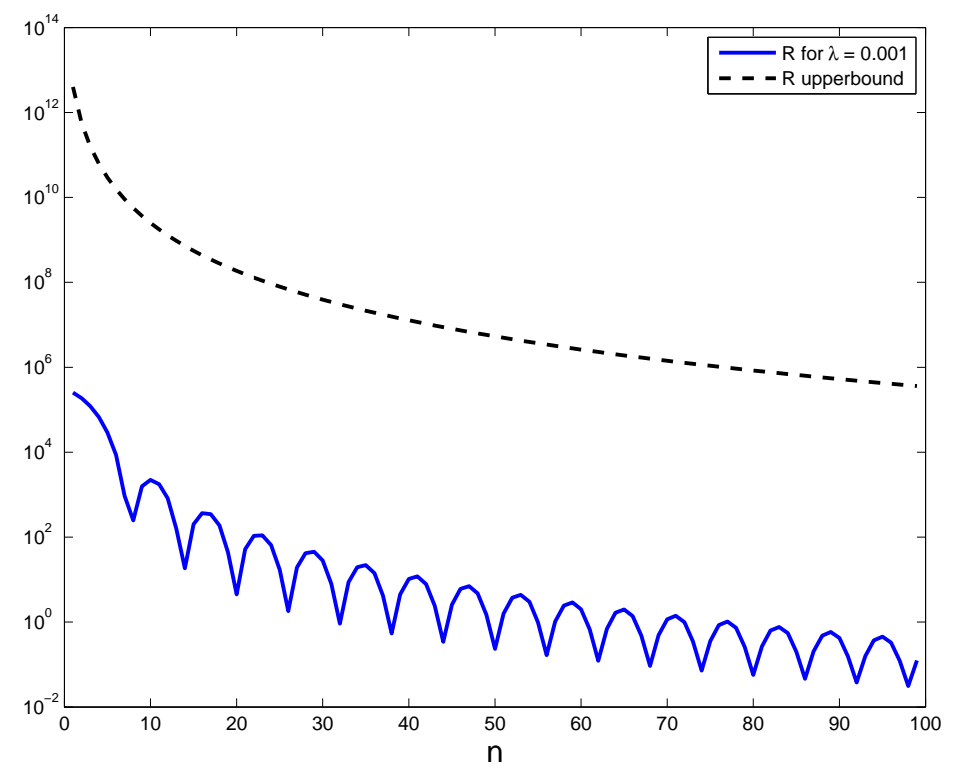

Figure 5 Semilog plot of the radius in function of $n$ for $\lambda=0.001$ and $\alpha=1.001 e^{i \frac{\pi}{3}}$, and of the upper bound (15) for the radius 